\title{
Evaluating a transdiagnostic acceptance and commitment therapy psychoeducation intervention
}

\author{
Jessica Cartwright $^{1}$ and Nic Hooper ${ }^{2 *}$ \\ ${ }^{1}$ Hywel Dda University Health Board, Wales, UK \\ ${ }^{2}$ University of the West of England, Coldharbour Lane, Bristol, UK
}

\begin{abstract}
Psychoeducation courses have gained some empirical support as effective early intervention strategies. Many of these courses reflect traditional cognitive behaviour therapy (CBT) thinking but psychoeducation courses based on other approaches are beginning to emerge. One such course, 'ACTivate Your Life', is based on acceptance and commitment therapy (ACT). The aim of this preliminary investigation is to evaluate a four-session (eight-hour) ACT psychoeducation intervention delivered within the Abertawe Bro Morgannwg University (ABMU) Health Board. Participants were invited to complete four outcome measures (assessing depression, anxiety, selfesteem and life satisfaction) and two process measures (assessing mindfulness selfefficacy and psychological flexibility) at pre- and post-intervention. Statistical analysis indicated that participants' scores across each of the measured domains showed highly significant changes. These results suggest that a brief psychoeducation ACT course may be useful in helping people in need of early psychological intervention, and that further research is now needed to provide a definitive evaluation of its effectiveness.
\end{abstract}

Key words: ACT, psychoeducation

\section{Introduction}

The Welsh model for the provision of mental health services distinguishes a number of layers or 'tiers' (Policy Implementation Guide, 2012). The first (least intensive) layer ('Tier 0') involves broad-based, low-cost interventions. In the Abertawe Bro Morgannwg University (ABMU) Health Board, such services are provided through an integrated primary-care related programme known as 'Living Life Well' (LLW), which has a self-referral pathway and no waiting lists. In order to meet the demands of such service provision, the Tier 0 programme prioritizes high volume and low intensity interventions, such as psychoeducation courses, delivered live to groups of participants.

The delivery of psychoeducation courses at Tier 0 accords with the suggestion by the National Institute for Health and Care Excellence (NICE, 2011) that, for individuals

\footnotetext{
*Author for correspondence: Dr Nic Hooper, University of the West of England, Coldharbour Lane, Bristol BS16 1QY, UK (email: Nic.hooper@uwe.ac.uk).
} 
presenting with mild and self-limiting symptoms, psychoeducation should be considered before the use of complex assessment and treatment strategies. Such interventions may provide effective help that will remove the need for referral to higher tiered services. For a number of years the LLW programme has offered a well-established and well-supported (see Burns et al., 2016) psychoeducation CBT-based course called Stress Control (White, 2010). However, psychoeducation courses based on other therapeutic approaches are now beginning to emerge. One such course, 'ACTivate Your Life' (AYL), is now being used in several Health Boards in Wales and has been incorporated by ABMU into their LLW programme. AYL is based on acceptance and commitment therapy (ACT), a third-wave CBT model that has been shown to be effective with a wide range of mental health and physical health conditions (see Hooper and Larsson, 2015). ACT involves helping clients to clarify valued directions, whilst also equipping them with acceptance and mindfulness skills designed to help them navigate their way through the unwanted thoughts and feelings that are part and parcel of being a human being. In short, ACT aims to help clients become more psychologically flexible, which means accepting things that they cannot change or control whilst maintaining a commitment to act in accordance with personal values.

This paper reports a preliminary community-based service evaluation of the AYL course delivered within the ABMU Health Board. It analyses participants' scores on a number of clinical measures administered immediately before and immediately after a four-week, eight-hour intervention. It examined changes in these measures to determine whether participants showed significant improvements over the course of the AYL intervention.

\section{Method}

The current paper presents the evaluation data obtained from 12 AYL courses delivered across the ABMU Health Board. The course was advertised primarily by poster and leaflet distribution in GP practice surgeries and through other health departments and community venues. In total, 243 individuals $(63 \%$ female, $94 \%$ white British, with an average age of 46 years) attended session 1 . Where possible, participants completed the standard set of evaluation measures before the first session commenced (time 1) and at the end of the final session (time 2).

\section{Outcome measures}

Four outcome measures were used to assess depression (PHQ-8: Patient Health Questionnaire 8; Cronbach's alpha, 0.91), anxiety (GAD-7: Generalized Anxiety Disorder 7 scale; Cronbach's alpha, 0.93), self-esteem (Rosenberg Self-Esteem Questionnaire; Cronbach's alpha, 0.89) and general life satisfaction (a single-item measure).

\section{Process measures}

Two process measures were used to assess mindfulness-based self-efficacy (MSES-R: Mindfulness-based Self Efficacy Scale Revised; Cronbach's alpha, 0.84) and psychological 
flexibility (AAQ-II: Acceptance and Action Questionnaire, second version; Cronbach's alpha, $0.92)$.

\section{Intervention}

AYL was developed by Professor Neil Frude. It is mainly didactic in nature, but also includes many non-interactive activities. The course consists of four two-hour sessions delivered over four consecutive weeks. The sessions are entitled: ACT 1 - You Are Not Your Mind; ACT 2 - Facing Up to Life; ACT 3 - Being Mindful; and ACT 4 - Living Wisely, Living Well. Participants are given a two-page handout that provides a summary of the content of the week's session and a four-page worksheet that describes a number of suggested home activities relating to the week's presentation. Courses are delivered in community-based settings across the Health Board by two or three presenters who have received the relevant presenter training.

\section{Results}

From the 243 people that agreed to take part in the study at pre-intervention, 109 participants completed the measures at post-intervention (a 55\% drop-out rate). This high drop-out rate, which is discussed in detail later in the article, is accounted for in the Results section via an intent-to-treat analysis, using the 'last observation carried forward' method. In other words, participants' scores at pre-intervention duplicated as their post-intervention score. Table 1 shows the mean scores across each of the measures at the beginning and the end of the course, for treatment completers and for all participants; the table seems to indicate improved scores across each measure.

Table 2 shows the $F$-values and effect sizes following data analysis using repeated measures ANOVAs; the table indicates, for participants who completed treatment, highly significant improvements in levels of depression, anxiety, self-esteem, life satisfaction, mindfulness self-efficacy and psychological flexibility from pre- to post-intervention. These significant improvements were maintained when including all participants, i.e. accounting for drop-out using the intent-to-treat analysis.

Table 1. Pre- and post-mean scores and standard deviations for all psychometric measures, for treatment completers and all participants

\begin{tabular}{lccccr}
\hline & \multicolumn{2}{l}{ Completers } & & \multicolumn{2}{l}{ All participants } \\
\cline { 2 - 3 } \cline { 5 - 6 } & Time 1 & Time 2 & & Time 1 & \multicolumn{1}{c}{ Time 2 } \\
\hline PHQ-8 & $13.96(6.32)$ & $8.34(5.27)$ & & $13.22(6.75)$ & $10.71(6.65)$ \\
GAD-7 & $12.15(5.98)$ & $7.19(5.12)$ & & $11.77(6.41)$ & $9.55(6.42)$ \\
RSE & $13.59(6.43)$ & $17.33(5.98)$ & & $14.51(6.71)$ & $16.18(6.55)$ \\
Life satisfaction & $4.51(2.22)$ & $6.05(1.97)$ & & $4.55(2.43)$ & $5.23(2.48)$ \\
MSES-R & $23.72(8.73)$ & $28.31(9.49)$ & & $24.50(9.29)$ & $26.55(9.72)$ \\
AAQ-II & $46.32(12.33)$ & $39.23(11.60)$ & & $45.13(13.48)$ & $41.97(13.37)$ \\
\hline
\end{tabular}


Table 2. F-values and effect sizes for treatment completers and for all participants

\begin{tabular}{lcclll}
\hline & \multicolumn{2}{c}{ Completers } & & \multicolumn{2}{c}{ All participants } \\
\cline { 2 - 3 } \cline { 6 - 6 } \cline { 5 - 6 } & $F$-value & Effect size & & $F$-value & Effect size \\
\hline PHQ-8 & 134.08 & 0.55 & & 79.79 & 0.25 \\
GAD-7 & 103.79 & 0.49 & & 68.11 & 0.22 \\
RSE & 54.03 & 0.33 & & 42.53 & 0.15 \\
Wellbeing & 65.72 & 0.39 & & 48.79 & 0.17 \\
MSES-R & 32.88 & 0.23 & & 28.28 & 0.10 \\
AAQ-II & 48.55 & 0.31 & & 39.08 & 0.14 \\
\hline
\end{tabular}

\section{Discussion}

This paper compared individuals' scores on clinical and wellbeing measures taken just before and immediately after they had participated in a four-week ACT-based psychoeducation course. The analysis indicated that individuals' scores on a range of outcome measures showed highly significant changes from time 1 to time 2 . Significant changes were also found on process measures of mindfulness-based self-efficacy and psychological flexibility. Thus it appears likely that the AYL course had a significantly positive clinical impact. Such benefit from a low-intensity psychological intervention reinforces the message given by NICE guidelines (2011) and may be regarded as especially relevant given the high prescription rates for psychotropic medication in Wales (Roberts, 2016).

Despite these positive findings, there are aspects to this research that may limit its impact. For example, the 'open walk-in' nature of the course prevented the recruitment of a suitable control group. Having such a control group would remove the possibility that psychological placebo powered outcome. Additionally, the anonymity of participants prevented the collection of follow-up data, which means that we cannot know if the intervention had long-lasting impact.

Most importantly, however, although attrition in psychoeducation interventions is generally high, there are a number of reasons that drop-out in the current context may have been even higher than other comparable studies (Burns et al., 2016). Firstly, people on the courses were informed that completing the questionnaires was not mandatory. This would have had been particularly problematic at post-intervention because completion of the questionnaires at that time-point would have meant hanging around at the venue after session 4 had finished. Indeed, it is important to note that despite drop-out from the study being high, drop-out from the course, as recorded by the presenters counting attendance, was much less severe (around $35 \%$ ). Another potential reason for the high drop-out rate is that, at pre-intervention, some participants who completed the questionnaires may have attended for the first session only in order to support a loved one, rather than for their own benefit. Finally, given that the people attending this course were probably unfamiliar with clinical research, it is possible that many were not able to remember the anonymous codes that they created at pre-intervention. Indeed, at post-intervention 33 completed questionnaire sets could not be matched to pre-intervention data.

It is important to make these points because they provide an argument that factors other than the content of the course may have impacted drop-out. It is also important, however, 
to acknowledge that the attrition rate recorded in the current study may be valid, i.e. high drop-out from the AYL course may be a 'real' phenomenon. Rather than this finding being perceived as negative, the authors of the current study believe that it could inform future research. In other words, future studies could investigate why people dropped out of the intervention and therefore develop ways to improve retention, and they could even conduct moderation analyses to determine if certain types of people at pre-intervention are better suited to this type of therapeutic delivery format.

Given the community-based open access nature of AYL, its potential cost-effectiveness and the highly significant changes observed in this service evaluation, together with encouraging qualitative feedback obtained from participants, a well-controlled study would appear to be warranted. Such a study would include a comparison control group (such as Stress Control), collect follow-up data and more carefully investigate attrition.

\section{Main points}

(1) An ACT-based psychoeducation intervention appears to be clinically useful as an early intervention strategy.

(2) Future research, which needs to include a control group and the collection of follow-up data, may wish to explore the high attrition rate found in this investigation.

\section{Acknowledgements}

None.

\section{Ethical statement}

The authors assert that the all procedures contributing to this work comply with the ethical standards of the relevant national and institutional committees of human experimentation and with the Helsinki Declaration of 1975 and its most recent revision.

\section{Conflicts of interest}

Jessica Cartwright and Nic Hooper have no conflicts of interest with respect to this publication.

\section{Financial support}

This research received no specific grant from any funding agency, commercial or not-for-profit sectors.

\section{Recommended further reading}

Harris R (2009). ACT Made Simple: An Easy to Read Primer on Acceptance and Commitment Therapy. Oakland, CA: New Harbinger.

Hooper N, Larsson A (2015). The Research Journey of Acceptance and Commitment Therapy (ACT). London: Palgrave Macmillan. 
White J (2010). Treating Anxiety and Stress: A Group Psycho-Educational Approach Using Brief CBT. Chichester: John Wiley \& Sons.

\section{References}

Burns P, Kellett S, Donohoe G (2016). 'Stress Control' as a large group psychoeducational intervention at Step 2 of IAPT Services: acceptability of the approach and moderators of effectiveness. Behavioural and Cognitive Psychotherapy 44, 431-443.

Hooper N, Larsson A (2015). The Research Journey of Acceptance and Commitment Therapy (ACT). London: Palgrave Macmillan.

National Institute for Health and Clinical Excellence (NICE) (2011). Common mental health problems: identification and pathways to care [CG123]. London: NICE.

Psychological Therapies in Wales: Policy and Implementation Guidance (2012). Cardiff: Welsh Government.

Roberts H (2016). Research Briefing: Misuses of Prescription and Over-the-Counter Medications. Cardiff: National Assembly for Wales.

White J (2010). Treating Anxiety and Stress: A Group Psycho-Educational Approach Using Brief CBT. Chichester: John Wiley \& Sons.

\section{Learning objectives}

(1) To acknowledge the potential of psychoeducation interventions at a Tier 0/ foundation level.

(2) To be introduced to a new psychoeducation course based on acceptance and commitment therapy (ACT) called 'ACTivate Your Life'.

(3) To acknowledge the effectiveness of 'ACTivate Your Life'.

(4) To consider the implications and limitations of this service evaluation.

(5) To see the argument that future research is needed to further establish the effectiveness of the course. 